\title{
Émigrer con gusto : les émigrés italiens et leur contribution à l'économie européenne aux XVII et $\mathrm{XVIII}{ }^{\mathrm{e}}$ siècles
}

Migrating con gusto: Italian migrants and their contribution to European material culture in the seventeenth and eighteenth centuries

\section{Giorgio Riello}

\section{(2) OpenEdition}

\section{Journals}

Édition électronique

URL : http://journals.openedition.org/dht/878

DOI : $10.4000 /$ dht.878

ISSN : 1775-4194

Éditeur :

Centre d'histoire des techniques et de l'environnement du Cnam (CDHTE-Cnam), Société des élèves du CDHTE-Cnam

\section{Édition imprimée}

Date de publication : 1 juin 2008

Pagination : $43-57$

ISBN : 978-2-95-30779-1-9

ISSN : 0417-8726

Référence électronique

Giorgio Riello, «Émigrer con gusto : les émigrés italiens et leur contribution à l'économie européenne aux xVII et xVIII siècles », Documents pour I'histoire des techniques [En ligne], 15। $7^{\text {er }}$ semestre 2008, mis en ligne le 19 octobre 2010, consulté le 08 septembre 2020. URL : http://journals.openedition.org/dht/ 878 ; DOI : https://doi.org/10.4000/dht.878 


\title{
Émigrer con gusto: les émigrés italiens et leur contribution à l'économie européenne aux xVII ${ }^{\mathrm{e}}$ et $\mathrm{xVIII}{ }^{\mathrm{e}}$ siècles*
}

\author{
Giorgio Riello \\ Global history and culture centre, \\ Université de Warwick
}

\begin{abstract}
Résumé
Le rôle des migrants italiens dans la diffusion du « goût italien » est bien connu dans au Xxe siècle : le design, la mode et le « made in Italy» sont aujourd'hui largement appréciés. Mais on sait moins que la relation entre esthétique et culture italienne est portée par une lingue histoire, depuis la Renaissance. Ce lien s'est maintenu auX XVIII et XVIII' siècles quand l'économie italienne décline et se marginalise. Les migrants jouent un rôle clé pour conforter le goût italien dans les arts décoratifs, mais aussi dans la production de biens de consommation allant de la verrerie aux comestibles. Dans cet article, on examine deux cas : les ouvriers du stuc, issus du Tessin, une aire limitrophe entre I'Italie et la Suisse ; les détaillants de nourriture italienne à Londres dans la deuxième moitié du XVIII siècle. Si ces deux cas révèlent des différences structurelles, ils expriment aussi des traits communs qui tiennent à la manière dont ces communautés ont utilisé l'identité italienne et leurs liens sociaux pour soutenir leur cause personnelle et, à la fois, promouvoir l'esthétique et les codes de civilité italiens à travers l'Europe.
\end{abstract}

Résumés et mots clés en anglais sont regroupés en fin de volume, accompagnés des mots clés français.

$\mathrm{L}^{2}$ a part de l'Italie dans la formation des économies du monde moderne est un sujet encore largement débattu. Les difficultés que cette nation a rencontrées lors de l'élaboration d'une notion solide d'identité nationale contrastent avec le succès de «l'exportation » du style italien. Au cours du xx siècle, l'adjectif « italien » en est venu à représenter un grand nombre d'idées concernant la culture de consommation, le goût et le style. Ces idées ont forgé le système économique italien $d$ 'après-guerre et ont construit une image complexe des produits, du style et du mode de vie italiens - la dolce vita. Starbucks parle italien, offre un grand choix de cafés, depuis le cappuccino jusqu'au macchiato en

* Cet article a été traduit à partir de l'anglais par Marie-Anne Michaux. J'aimerais remercier Liliane Pérez de m'avoir invité à présenter une première version de cet article au colloque «Minorités et circulations techniques du Moyen Âge à l'époque moderne», tenu au Conservatoire national des arts et métiers le 4 juin 2004 passant par le latte ${ }^{1}$. De la même façon, la mode et le design parlent italien, depuis les designers bien connus comme Alessi, Armani, Valentino, Gucci et Ferragamo jusqu'au concept plus large du « made in Italy $»^{2}$.

$\|$ mest nécessaire de me référer au présent afin de comprendre les problèmes que rencontre la reconstruction de la participation de l'Italie dans I'histoire étrangère. L'idée qu'il existe un « produit italien » et que ses caractéristiques soient le résultat du sens du style des Italiens est plus qu'un simple cliché et s'étend au-delà de l'Italie. Le succès du « made in Italy »au xxe siècle n'est pas un phénomène

\footnotetext{
1 Ceux-ci sont le sujet d'une recherche en cours conduite par Jonathan Morris de I'université de Hertfordshire et financée par le Cultures of consumption project sur : "The Cappuccino conquests : a transnational history of Italian coffee ». 2 Fortis M., II Made in Italy, Bologne, II Mulino, 1998 ; Settembrini L., Made in Italy?: 1951-2001, Milan, Skira, 2001.
} 
national mais a acquis une force certaine à travers la reconnaissance internationale. Le lien entre la production nationale et les ventes internationales est le rôle de l'émigrant. II est en effet possible de soutenir que ce que I'Italie a exporté avec le plus de succès fut sa population. Á l'exception de I'Irlande, aucun autre pays européen n'a produit autant d'émigrants sur une période de plus d'un siècle. Les chiffres impressionnent : déjà en 1891, plus d'un million et demi d'Italiens vivait à l'étranger ${ }^{3}$.

Il a souvent été dit que l'on devait à l'émigration de millions d'Italiens la puissance de l'identité italienne à l'étranger. Les Italiens n'ont pas fait que "s'exporter ». Avec eux sont aussi venus les traits distinctifs de la vie italienne: dans les années 60 les designs à la pointe de la mode des appareils ménagers étaient vendus autour du monde en s'appuyant sur un réseau d'émigrés italiens ${ }^{4}$. Cela est valable pour les voitures, les vêtements, les montures de lunettes, les meubles et les produits alimentaires. Plus que n'importe quelle autre appellation nationale, le label « made in Italy » renferme la relation entre qualité et provenance nationale. ॥ transcende le produit individualisé pour identifier un ensemble de produits variés. Leur « qualité », non pas technique ou technologique au sens strict, dérive de la capacité de ce label assez évanescent, à donner une image du bon goût et du design. II a beaucoup été écrit sur la façon dont le système de production italien est alimenté par la création et la perpétuation de ces qualités de production à travers le secteur industriel, les petites entreprises et les solutions de production semi-artisanales ${ }^{5}$. Les écrits sont plus rares en ce qui concerne la trajectoire historique de l'Italie comme créateur de « bon goût » ${ }^{6}$.

\footnotetext{
3 II existe une abondante littérature sur les migrations italiennes des $x \mid x^{e}$ et $x x^{e}$ siècles. Un outil bibliographique utile est : Sponza L., Italy, Oxford, Clio Press, 1986, plus particulièrement pp. 118-128. 4 Gomellini M., I/ commercio estero dell'Italia negli anni sessanta specializzazione internazionale e tecnologia, Rome, Banca d'Italia, 2004.

5 Goodman E. et Bamford J. éd., Small firms and industrial districts in Italy, Londres, Routledge, 1989; Quadrio Curzio A., Fortis M. éd., Complessità e distretti industriali: dinamiche, modelli, casi reali, Bologne, II Mulino, 2002. Pour des études plus générales voir : Amatori F. et Colli A., Impresa e industria in Italia: dall'Unità a oggi, Venice, Marsilio, 1999; Colli A., I volti di Proteo: storia della piccola impresa in Italia nel Novecento, Turin, Bollati Boringhieri, 2002.

6 Le label même " made in Italy " a été inventé au début des années 50 quand la compétition contre la mode française (qui avait traditionnellement dominé le marché international depuis le xvIII siècle) devint intense.
}

Dans cet article, on souhaiterait « partir à reculons » depuis le $x x^{e}$ siècle jusqu'au début de la période moderne en s'attachant à l'analyse des communautés italiennes à l'étranger et leur réussite dans ce que je qualifierais de « goût commercial ». Il faut souligner que dans la période étudiée seulement quelques milliers d'Italiens résidaient hors des frontières de l'Italie. L'argument principal de cet article est qu'entre 1600 et 1800 les Italiens ne sont pas globalement aussi « actifs » que pendant la Renaissance. Ce qu'ils exportent sont leurs aptitudes visuelles, tactiles et auditives appliquées aux arts décoratifs (la peinture mais aussi les gravures et les produits de luxe tels que le verre, la majolique et la joaillerie), à l'architecture, la musique et la « haute cuisine ». L'influence des Italiens dans la sphère particulière du design, du style et du goût mêle les talents pratiques, le savoir et les modèles culturels d'une manière qui peut être reliée à l'ascendance, commercialement bien inspirée, du design italien et du « made in Italy » du xx siècle. La complémentarité entre fabrication et services, tels qu'ils sont compris dans la notion du goût et du design italien, est étudiée à travers deux exemples : les stuccatori italiens travaillant en Europe centrale et en Europe du Nord aux XVII et $\mathrm{XVIII}$ e siècles, et le monde des restaurateurs italiens de Londres dans la seconde moitié du XVIII siècle.

\section{Individus, groupes et réseaux}

Les « communautés » médiévales de marchands italiens vivant dans les piazze d'affari les plus importantes d'Europe ont été largement étudiées par les historiens intéressés dans la cartographie du marché ainsi que dans la reconstruction de l'articulation complexe des interconnections financières entre les grands établissement commerciaux et bancaires de Toscane et de Lombardie et leurs succursales sur le Continent, plus particulièrement en Europe du Nord ${ }^{7}$. « Les époux Arnolfini » peint par Van Eyck en 1434 et censé célébrer

7 Par exemple, Lombard Street à Londres, doit son nom à la communauté de marchands du nord de l'Italie vivant ici à la fin du Moyen Âge. Crinó A. M., «Italiani in Inghilterra dal trecento ai nostri giorni », Archivio Storico Italiano, 126, 1968, p. 363. Voir aussi Bayard F., "Les Bonvisi, marchands banquiers à Lyon, 1575-1629 », Annales ESC, 16, hiver 1971, pp. 1234-1267 et Marietti M., "Les Rucellai en France : marchands, humanistes, diplomates », dans Fontana A., Marietti M., Pozzi M., éd., La circulation des hommes et des œuvres entre la France et l'Italie de la Renaissance, Paris, Presses Sorbonne nouvelle, 1992, pp. 39-60. 
le mariage du marchand italien Giovanni Arnolfini avec sa fiancée italienne Giovanna Cenami, est sans doute I'un des portraits les plus connus d'un marchand italien de la première Renaissance exerçant à Bruges ${ }^{8}$. Les banquiers et financiers internationaux, comme Arnolfini, avaient de fortes connexions, dans leur patrie comme à l'étranger grâce à un réseau composé d'autres marchands, de serviteurs et messagers ${ }^{9}$. Ils profitaient d'un statut et d'une reconnaissance sociale dans leurs communautés d'accueil et s'intégraient mieux aux organisations civiles locales que les autres Italiens circulant en Europe: étudiants, pèlerins et membres du clergé, mais aussi des exilés politiques et des vagabonds ${ }^{10}$. Pour ces derniers, leur présence à l'étranger était souvent limitée dans le temps et peut difficilement être réduite à la notion moderne de « migration» ${ }^{11}$.

Davantage que des émigrants, les personnes comme Arnolfini sont reconnues comme des agents internationaux du développement économique. Les communautés de marchands agissent comme des intermédiaires dans l'importation et l'exportation, et tiennent, à la fin du Moyen Âge, une place centrale

8 Cette peinture est maintenant à la National Gallery, Londres, NG186.

9 Voir par exemple: Comba R., «Emigrare nel Medioevo. Aspetti ecomico-sociali della mobilità geografica nei secoli XI-XVI », dans Comba R., Piccinni G. et Pinto G. éd., Strutture familiari, epidemie, migrazioni nell'Italia medievale, Naples, ESI, 1984, pp. 45-74; Rossetti G. éd., Dentro la città. Stranieri e realtà urbana nell'Europe dei secoli XII-XVI, Naples, Gisem-Liguori, 1989; et Del Treppo M. éd., Sistema dei rapporti ed elites economiche in Europa (secoli XII-XVII), Naples, Gisem-Liguori, 1994.

10 Pizzorusso G., «I movimenti migratori in Italia in antico regime», dans Bevilacqua Piero, De Clementi A. et Franzina E. éd., Storia dell'emigrazione Italiana, Rome, Donzelli Editore, 2001, p. 6.

11 Sur l'idée des forestiero et leur rôle dans la formation des identités nationales, voir : Petti Balbi G. éd., Comunità forestiere e 'nationes' nell'Europa dei secoli XIII-XVI, Naples, Liguori, 2001, en particulier l'article par Enrico Basso sur «Note sulla comunità genovese a Londra nei secc. XIII-XVI», pp. 249-268. Aussi importants, les articles par Michele Cassandro, «l forestieri a Lione nel '400 e '500: la nazione fiorentina», dans Rosetti G., éd., Dentro la città, op. cit. note 9, pp. 151-162 et Gauvard C., L'étranger au Moyen Âge. XXXe congrès de la SHMES, Paris, Publications de la Sorbonne, 2000. Il est aussi difficile de parler de marchands " italiens », surtout au Moyen Âge, quand les provenances régionales influencent les modèles de résidence. Dans le cas du Londres de la fin du xive siècle, par exemple, les Florentins et les Lucchesi tendent à s'installer de façon permanente dans la capitale anglaise, plus que les marchands vénitiens, plus transitoires. Voir Bradley H. L., « The Italian community in London, c. 13501450 », thèse de Ph.D., Université de London, 1992. dans le dialogue technologique entre l'Italie et l'Europe du Nord ${ }^{12}$. Les historiens d'art soulignent le rôle des élites marchandes dans la transmission du goût et du style depuis l'Europe du Sud, en particulier depuis les cours italiennes, les États de la Papauté et les principaux centres de production artistique de la péninsule. Le succès international de la plupart des motifs décoratifs, de l'architecture, mais aussi de la mode vestimentaire et des objets de consommation ostentatoire, est dépendant des Italiens circulant en Europe autant que des étrangers voyageant en Italie à la recherche d'une ancienne culture, d'expériences pieuses et d'idéaux de la Renaissance.

Le chevauchement entre migration et talents artistiques est tellement ancré dans la mentalité italienne que jusqu'au début $d u$ XIx siècle les émigrants italiens se désignent eux-mêmes par le nom « d'artiste » pour signifier leur appartenance au type idéal de l'émigré peintre, sculpteur, décorateur, maçon ou architecte ${ }^{13}$. Si nous nous limitons à l'Angleterre ${ }^{14}$, le nombre d'Italiens illustres quittant le pays pour l'Albion est impressionnant, allant des figures littéraires telles que Poggio Bracciolini et Baldassare Castiglione durant les Quattrocento et Cinquecento, jusqu'au literati du XVIII et XVIII siècles comme Scipione Maffei, Paolo Rolli et Giuseppe Baretti15. Le portrait groupé de Francesco Bartolozzi, Giovanni Battista Cipriani et Agostino Carlini par John Francis Rigaud (1777) permet d'entrevoir la contribution de l'Italie à l'establishment intellectuel anglais de la seconde moitié du XVIII ${ }^{\mathrm{e}}$ siècle ${ }^{16}$.

12 Postan M. M., «Spread of techniques : Italy and the economic development of England in the Middle Ages », Journal of economic history, 11-4, 1951, pp. 339-346.

13 Gabaccia Donna E., "Class, exile, and nationalism at home and abroad: the Italian Risorgimento », dans Gabaccia Donna E. et Ottanelli Fraser M. éd., Italian workers of the world: labor migration and the formation of multiethnic states, Urbana et Chicago, University of Illinois Press, 2001, p. 26.

14 II existe aussi une littérature expansive sur les Italiens dans la France du début de la période moderne. Voir : Dubost J.-F., « L'étranger dans la France d'Ancien Régime : ambiguïtés d'une perception », dans Jessenne J.-P. éd., L'Image de l'autre dans l'Europe du Nord-Ouest à travers I'histoire, Villeneuve-d'Asq, Centre d'histoire de la région du Nord et de l'Europe du NordOuest, 1996, pp. 33-42 ; id., La France italienne : xVIe-xvI siècle, Paris, Aubier, 1997. Pour une bibliographie étendue, voir : Heller $\mathrm{H}$., Anti-Italianism in sixteenth-century France, Toronto et Londres, University of Toronto Press, 2003.

15 Graf A., L'Anglomania e I'Influsso Inglese in Italia nel Secolo XVIII, Turin, E. Loescher, 1911, pp. 53, 59, 390.

16 Ce portrait a été peint comme un pendant à un triple portrait similaire de Reynolds, Chambers et Wilton. West S., "Xenopho- 
Les communautés commerciales, le rôle joué par des individus doués et de grands artistes, l'élan général de la vie économique de la péninsule italienne à la fin du Moyen Âge et à la Renaissance sont des facteurs expliquant pourquoi la technologie et les inventions italiennes, mais aussi les formes de consommation et les pratiques commerciales et financières, se sont répandues à travers toute l'Europe. Ces facteurs sont des thèmes traditionnels de I'histoire économique qui s'attache à expliquer l'ascendant de l'Europe du Nord et du monde atlantique en mettant en valeur la contribution de la péninsule italienne dans l'économie et la vie culturelle européennes. À l'inverse, la période entre la stagnation économique de l'Italie du xvII siècle (moment de peste, misère et soumission politique, immortalisé par Alessandro Manzoni dans ses Promessi Sposi) et le Risorgimento du début du XIXe siècle apparaît comme une page presque vierge de l'histoire de l'Italie. À cette époque, le pays se retire du devant de la scène pour rester dans les coulisses, bénéficie d'une faible croissance économique et, plus généralement, participe peu aux innovations dans le domaine de l'économie et de la vie culturelle européennes. Cette vision noire a récemment été rejetée par de nombreux historiens qui, sans nier les résultats modestes de l'économie italienne du XVII et $x V I I I^{e}$ siècles, expriment des doutes quant à la position marginale que l'Italie a tenue dans la vie économique européenne ${ }^{17}$.

Cette relecture est importante puisqu'elle corrige les écrits sur le déroulement historique qui séparaient

bia and Xenomania : Italians and the English Royal Academy », in West S. éd., Italian culture in Northern Europe in the eighteenth century, Cambridge, Cambridge University Press, 1999, p. 123.

17 Salvatore Ciriacono, par exemple, soutient qu'au xvi siècle, I'Italie expérimente une expansion de la production des biens de luxe, plus particulièrement des textiles de haute qualité. Voir Ciriacono S., "Mass consumption goods and luxury goods : the de-industrialisation of the Republic of Venice from the sixteenth to the eighteenth century », dans Van de Wee Herman éd., Rise and decline of urban industries in Italy and in the Low Countries Late Middle Ages - Early modern times, Louvain, 1988, pp. 4161 ; id., "Les manufactures de luxe à Venise : contraintes géographiques, goût méditerranéen et compétition internationale (XIV $-x v l^{e}$ siècles) », dans Les Villes et la transmission des valeurs culturelles au bas Moyen Age et aux Temps Modernes, Bruxelles, Crédit Communal, Collection Histoire, 1996, pp. 235-251; id., «Economie urbane e industria rurale nell'Italia del Cinque e Seicento: riconversione o stagnazione? », Rivista storica italiana, 113-1, 2001, pp. 5-35. Voir aussi I'approche novatrice de Carlo Marco Belfanti « Guilds, patents, and the circulation of technical knowledge : northern Italy during the early modern age », Technology and culture, 45-3, 2004, pp.569-589. la Renaissance italienne de I'histoire de l'Italie moderne (post 1800). Cet article ne prétend pas offrir une analyse poussée de tels problèmes historiographiques mais considère simplement le rôle que ces communautés italiennes particulières jouèrent dans la vie européenne ${ }^{18}$. Ainsi qu'il a été dit dans l'introduction, cette étude suggère que I'Italie continue, aux $x \|^{\mathrm{e}}{ }^{\text {et }} \mathrm{xvII^{ \textrm {e } }}$ siècles, à être un point de référence important dans les choix esthétiques, artistiques et culturels hors de ses frontières. Le rôle de l'Italie, bâti à l'origine pendant sa

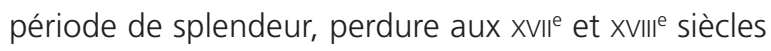
lorsque de nouvelles formes de consommation et une structure sociale de plus en plus dynamique viennent dominer la société européenne. Dès le $x x^{e}$ siècle, le style et le goût italien sont clairement reconnus à un niveau global. Ce résultat est obtenu non seulement grâce des produits et certaines pratiques sociales mais aussi grâce à des communautés d'Italiens qui transmettent ce qui sera appelé plus tard le « style italien ».

\section{Les stuccatori du Tessin}

L'un des meilleurs exemples de relation symbiotique entre communautés étrangères et popularisation du goût italien est le cas des stuccatori italiens. Les travailleurs du stuc italiens, ou stuccatori, ont été largement étudiés par les historiens d'art mais sont encore peu présents dans les travaux des historiens. Ce qui a été souvent observé c'est la concentration géographique de l'artisanat du stuc. Les travaux en stuc et en plâtre sont fréquemment utilisés dans $I^{\prime} I$ talie des $x V l^{e}, x V I I^{e}$ et $x V I I I^{e}$ siècles, et presque toutes les régions de la péninsule pouvaient se vanter de posséder quelques artisans particulièrement talentueux dans ce domaine. Mais c'est d'une aire géographique bien spécifique, le Tessin, que sont sortis un nombre sans précédent de stuccatori. Le canton du Tessin, dont la plus grande partie est aujourd'hui comprise à l'intérieur des frontières de la Suisse, est une petite région montagneuse qui a été pendant des siècles le limes entre les États italiens du Sud et l'Empire d'Europe centrale. Le Tessin est donc par tradition une zone de

18 Voir par exemple le travail d'Hélène Verin sur les Italiens dans les travaux de fortifications et l'étude de Pascal Brioist et al. sur les maîtres escrimeurs italiens. Vérin H., Entrepreneurs entreprises : histoire d'une idée, Paris, Presses universitaires de France, 1982 ; Brioist P., Drévillon H. et Serna P., Croiser le fer : violence et culture de l'épée dans la France moderne (XVI -XVIII siècle), Seyssel, Champ Vallon, 2002. 
passage entre Europe du Nord et Europe du Sud. Bien que catholique et italianophone, il a aussi été fortement influencé par l'Europe centrale. Les mouvements migratoires, du nord au sud et inversement, n'étaient pas rares et sont restés, jusqu'à une période récente, I'une des caractéristiques de cette région ${ }^{19}$.

Le trait le plus frappant dans I'histoire de cette région est peut-être sa créativité artistique qui a produit tout au long des siècles quantité d'architectes, peintres et sculpteurs bien connus. Les architectes romains Domenico et Giovanni Fontana sont deux frères nés à Melide, ainsi que leur neveu Carlo Maderno, I'architecte de la façade de la basilique Saint-Pierre. Francesco Borromini, sans doute le plus célèbre des architectes baroques de la Rome $d u x \|^{e}$ siècle, est né à Bissone et a été un élève de son compaesano Carlo Maderno ${ }^{20}$. Le Tessin, cependant, ne se limite pas à être la terre natale "d'artistes » célèbres. Entre le $x v l^{e}$ et le début du XIX siècle, un nombre sans égal de travailleurs hautement spécialisés et étonnamment mobiles, connus dans toute l'Europe pour leurs travaux en stuc et plâtre, sont issus de cette région.

J'ai utilisé le terme « communauté » pour identifier un groupe d'Italiens résidant et travaillant hors de leur pays. Le cas des artisans du stuc du Tessin représente, toutefois, un type particulier de communauté d'émigrants. Les communautés étrangères sont normalement définies comme des groupes d'individus dont la présence simultanée dans le même pays (étranger) engendre la création de productions spécialisées, de réseaux économiques et financiers mais aussi sociaux et culturels, et l'éventuel succès (ou échec) de leur contribution à l'économie locale. Cette définition souligne comment la singularité des stuccatori réside à la fois dans leur nature diachronique et dans la géographie polycentrique de leur " communauté ». Leur expérience de la migration n'est pas un premier acte d'installation dans une terre étrangère mais se fonde plutôt sur les mouvements continus de villes en villes à la recherche de commandes. Dans la vallée du Tessin certaines périodes de l'année se passent dans I'attente d'un nouveau travail, en particulier durant les

19 Merzario R., «Famiglie di Emigranti Ticinesi (Secoli XVII-XVIII) », Società e soria, 19-71, 1996, pp. 39-55. Voir aussi Lorenzetti L., « Migration, strategies économiques et réseaux dans une vallée alpine : le vale de Blenio et ses migrants (XIX $x^{\mathrm{e}}$ - début $x \mathrm{x}^{\mathrm{e}}$ siecle) », Schweizerische zeitschrift für geschichte, 49-1, 1999, pp. 87104

20 Donati U., Breve storia di artisti ticinesi, Bellinzona, A. Salvioli, 1936, pp. 85-95. mois d'hiver. C'est aussi le cas des longues périodes de guerre qui monopolisent les fonds aux dépens des décorations princières et des bâtiments publics. Si les communautés étrangères qui se transplantent hors de leur pays apportent avec elles un ensemble de talents spécifiques et uniques qu'elles mettent ensuite à profit là où ces talents sont rares, les travailleurs du Tessin répètent ce processus chaque fois qu'ils arrivent dans un nouveau village ou une nouvelle ville, en Allemagne, Tchécoslovaquie, Pologne ou Angleterre.

II s'agit d'un trait important de leur travail puisque cela ressemble davantage à une prestation possible qu'à une capacité personnelle et indépendante de produire ou de fabriquer un artefact. L'essentiel de leur talent n'est pas leur capacité à construire des plafonds élaborés, à partir de leurs connaissances des propriétés du stuc et des substances colorées, mais leur habileté à créer un résultat esthétique conforme au goût du jour et incarnant la dernière mode en termes de décoration et d'architecture. Le fait que les stuccatori du Tessin restent au sommet de leur profession pendant six ou sept générations montre comment cette communauté « diachronique » est capable de se reproduire à travers le temps dans un contexte qui ne vient pas des limites géographiques étroites de leur vallée d'origine mais du monde artistique et culturel de l'Europe du Nord aux $x V I I^{e}$ et $x V I I I^{e}$ siècles.

Les notions classiques de réseaux nationaux denses partageant les mêmes cultures, langage et talents, et liés par une expérience de la migration commune ne suffisent pas pour comprendre la nature et la permanence dans le temps de la communauté du Tessin. À un premier niveau cela signifie que structurer sa carrière autour d'un réseau de camarades artisans travaillant dans la même région n'est pas suffisant. La renommée des artisans du Tessin grâce à leurs travaux en stuc reste une caractéristique de leur identité pendant une période considérable, entre le $x v l^{e}$ et la fin du XVIII siècle. Il y a sûrement une dimension éthique dans leur travail. II ne s'agit pas simplement d'émigrer pour trouver un emploi mais aussi d'être capable d'assurer un commerce prospère pour les générations futures de jeunes hommes venant d'une vallée perdue des Alpes. Les apprentis sont recrutés au village lors des visites que les artisans font à leurs amis et familles (en particulier leurs femmes) pendant la morte-saison ou entre deux périodes de travail.

Giovan Battista Clerici (vers 1680-1739) était un stucateur actif en Allemagne et en Europe centrale durant les premières décades $d u x \mathrm{VIII}^{\mathrm{e}}$ siècle. II venait 
de Meride, un petit village du Tessin maintenant en territoire suisse. À tout juste dix-huit ans, il est engagé par Giovan Pietro Magni, un artisan en stuc expérimenté venant du même village. Son histoire, et celles de bien d'autres stucateurs de la Vallée, émerge des quelque deux mille lettres préservées aux Archives cantonales du Tessin à Bellinzona. Ces lettres étaient envoyées par les artisans de toute l'Europe aux Ortelli, une famille de notaires qui prenait soin des contrats de travail des artisans, ainsi que de leurs affaires personnelles, familiales et légales. Clerici était particulièrement précis dans l'envoi de nouvelles sur ses progrès mais aussi sur ses considérations personnelles. Après à peine quelques mois d'entraînement, il commente qu'il «a appris beaucoup pendant cette période » ajoutant que « durant l'hiver entier je n'ai pas perdu une heure et ai sculpté continuellement ${ }^{21}$ ». Il était employé au château de Würzburg en Allemagne du Sud et en I'espace de quelques années se déplaça de plusieurs centaines de kilomètres, travaillant avec différents maîtres, puis comme artisan indépendant.

Les stucateurs comme Clerici ne se voyaient pas comme appartenant à une plus vaste communauté mais se représentaient leur monde social et professionnel en termes de liens temporaires avec d'autres artisans du Tessin, dont les vies professionnelles, cela était bien compris, se croiseraient périodiquement. Du point de vue du jeune Clerici, il faisait avant tout partie d'un groupe d'artisans gyrovagues parlant une langue commune (le dialecte du Tessin), avec des amis et des liens familiaux communs dans leur terre natale. Une fois I'apprentissage terminé, il n'était pas difficile de trouver un emploi, bien que les commandes spécifiques soient rarement de longue durée. Quand un nouveau travail était recherché, les stucateurs savaient où aller et qui contacter. Les camarades artisans étaient le moyen le plus sûr et le plus simple d'envoyer des nouvelles au pays et les documents des Ortelli montrent qu'il était fréquent qu'un groupe d'hommes écrivent ensemble une lettre afin de réduire le coût de la livraison. Ils partageaient aussi une religion commune dans les pays austères et peu accueillants des états d'Europe centrale. Ainsi, le jeune Clerici écrit à son village quelques mois

21 "lo mi sono vanzato asai in questo tempo, in tutto l'inverno non ò perso un'ora e ò sempre lavorato dintaglio continovamente". Cité dans Martinolo G., "L'itinerario in terra tedesca dello stuccatore Giovan Battista Clerici di Meride », dans Aslan E. éd., Arte e artisti dei laghi lombardi. II. Gli stuccatori dal Barocco al Rococo, Côme, Tipografia Editrice Antonio Nosera, 1964, p. 305. après l'avoir quitté que « en ce qui concerne le corps je me porte bien, mais pour ce qui est de l'âme je me porte mal car je ne peux assister à la messe si ce n'est une fois tous les deux mois $22 »$.

Le cas de Clerici montre cependant comment des années de travail et d'expérience dans le métier, des voyages continus et des emplois abondants changent profondément la perception de sa position dans la profession. Après quinze ans de carrière, Clerici est capable de se placer dans le contexte d'un métier dont la continuité transcende sa propre existence. II est alors prompt à prendre des apprentis dans son propre village "pour leur apprendre tout ce que je sais ${ }^{23}$ ». Les apprentis arrivent sans aucune qualification mais peuvent apprendre rapidement. La langue commune était sûrement un avantage par rapport aux apprentis allemands, tchèques ou polonais. La nouvelle génération doit parler italien pour pouvoir travailler avec le nombre (substantiel) d'architectes venant du sud des Alpes pour construire les châteaux, villes et églises baroques des actuelles Autriche, Tchécoslovaquie, Pologne et Allemagne. Mais il existe aussi des raisons plus personnelles. À travers les apprentis italiens, la tradition peut continuer en reliant l'émigrant à ses propres racines. De plus, la reconnaissance sociale est un stimulant suffisant : transmission à la terre d'origine du bénéfice d'une carrière, création d'une richesse possible, source d'emploi pour le futur. Ce qui explique pourquoi l'un des apprentis de Clerici était un orphelin de son propre village ${ }^{24}$.

Dans bien des cas, la dimension régionale de ce groupe de travailleurs qualifiés est renforcée par des stratégies et des relations familiales. La famille d'Allio, par exemple, était une compagnia di lavoro formée dans les dernières années du XVII ${ }^{e}$ siècle de trois artisans apparentés: Giovanni Battista était le décorateur de la chapelle de l'orphelinat à Passavia et de l'église Saint Benoît à Niederaltaich en Bavière. Son fils Paolo s'occupait de la chapelle et de la résidence de la cour de Passavia et son autre fils Sebastiano travaillait sur I'église principale de Niederaltaich. Une autre entreprise familiale était conduite par les Bossi ${ }^{25}$. Giuseppe Antonio Bossi était renommé pour la qualité de son artisanat

22 "In riguardo al corpo sto bene, ma per l'anima malle, perchè non poso sentir messa se non una volta ogni doi mesi", ibid., p. 307.

23 "insegnarli quell tanto che so", ibid., p. 308.

24 Ibid, pp. 309-11.

25 Hermanin F., Gli Artisti in Germania, vol. 2. Gli scultori, gli Stuccatori, i cramisti, Rome, Libreria dello Stato, 1935, p. 23. 


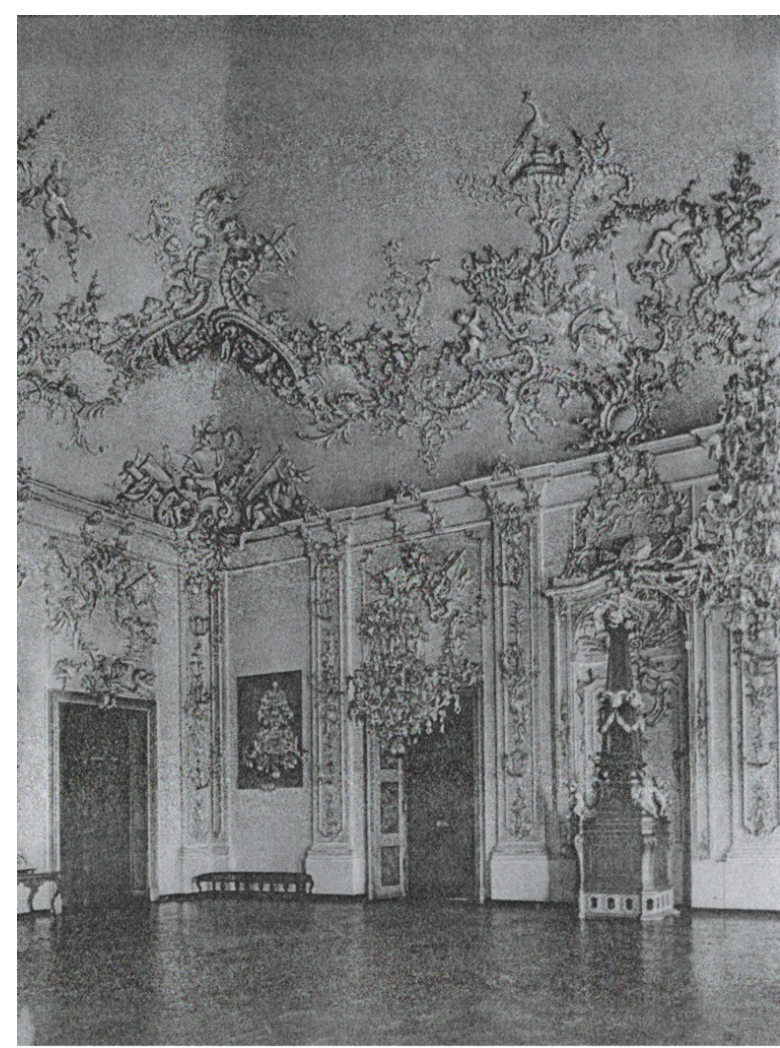

Fig. 1. Plafond par Giuseppe Antonio Bossi au Palais de Würzburg, Allemagne. (F. Hermanin, Gli Artisti in Germania, Rome, 1935, p. 23).

et connu dans toute l'Europe grâce à ses plafonds du palais de Würzburg (cf. fig. 1). Sa réputation a permis à ses frères Lodovico et Agostino de réussir dans la recherche d'un emploi et à la dynastie de continuer à travers un autre artiste de talent, son neveu Materno ${ }^{26}$. Dans d'autres cas, la force de I'association familiale pouvait être telle qu'elle imposait une marque ou un style de travail particulier. La famille Carlone, de Rovio, par exemple, a produit plus de quarante architectes, peintres, sculpteurs et stuccatori. Les stucateurs surtout étaient fameux pour leur style appelé « Carlonesco », à cause de la technique particulière de Giambattista Carlone avec de larges traits et un certain aspect d'inachevé. La famille profita de cette marque de fabrique qui resta efficace à travers l'Europe centrale pendant la majeure partie du XVIII et la première moitié du XIX siècle ${ }^{27}$.

Le talent qui caractérise les stucateurs était une qualité pré-requise que chacun d'entre eux devait acquérir durant un court apprentissage. II convient

26 Ibid., pp. 20-21.

$27 \mathrm{Ibid} .$, pp. 23-24. de souligner toutefois qu'il ne s'agissait pas d'un entraînement particulier et que l'apprentissage ne dévoilait aucun " secrets ${ }^{28}$. Les stuccatori s'exportaient, ainsi que leur production, non pas tant grâce à leur capacité de production originale que grâce à une sensibilité visuelle, artistique et esthétique partagée. À un premier niveau, cela signifie prendre part à un circuit fermé de compagnons artisans qui peuvent fournir des contacts et assurer la qualité globale du travail. Bien sûr, il existait une compétition avec les Allemands et autres stucateurs européens, mais l'identité et la « spécialité » des artisans italiens restaient un symbole de qualité supérieure. Les relations sont essentielles. Comme nous I'avons déjà précisé, les architectes devaient savoir quels artisans contacter et les meilleurs parmi ces derniers ont eu des carrières météoriques à travers I'Europe. Giovanni Battista Artari (né en 1660) et son fils Giuseppe (né en 1697) ont été engagés par Gibbs pour la décoration du splendide plafond de Saint-Martin-des-Champs à Londres (1722-1726) (cf. fig. 2) et de Saint-Pierre dans Vere Street dans le West End $(1724)^{29}$. II semble que les Artari étaient en phase avec le goût de l'époque et dans le ton des principes palladiens présentés dans l'ouvrage récemment traduit Quattro Libri dell'Architettura et des règles vitruviennes ${ }^{30}$. Ceci explique pourquoi ils décorèrent le plafond de Ditchley Park dessiné par Kent et le plafond de Stone Hall à Houghton Hall entre l'été 1726 et le printemps $1728^{31}$. Houghton Hall était la plus importante et la plus réputée des résidences de campagne de la première Angleterre géorgienne. Ce n'était pas seulement la résidence luxueuse du Premier

28 Selon Geoffrey Beard, s'il y avait un « secret », il s'agissait de savoir remplir les pores à la surface du plâtre avec de la gomme ou un encollage (une substance collante) dissout dans de l'eau. Le résultat était travaillé en rouleaux en forme de saucisse, appliqués sur la surface. Une fois sec, ce matériau était frotté avec du grès dur et poli comme du marbre. Beard G., Craftsmen and interior decorators in England 1660-1820, Edinbourg, John Bartholomew and Son, 1981, p. 35.

29 Summerson J., Georgian London, Londres, Penguin, 1945, pp. 90-93 (sur St.-Martin-in the-Fields) et pp. 79, 110 (sur St. Peter). Palumbo-Fossati suggère que le plafond de St.-Martin a été fait par Bagutti et Artari. Voir Palumbo-Fossati C., Figure ed aspetti poco noti dell'attività degli artisti ticinesi in paesi lontani, Agno, Arti Grafiche Bernasconi, 1974, p. 7.

30 Les Quattro Libri avaient justement été traduits entre 1716 et 1720 par I'architecte Giacomo Leoni. Le succès de ces ouvrages ont assuré à Leoni certains des travaux les plus importants d'Angleterre tels que Lyne Park dans les années 1720 et Clandon Park dans les années 1730 .

31 Murray P., "Architettura inglese e stuccatori italiani », dans Aslan E. éd., Arte e artisti dei laghi, op. cit. note 21, p. 318. 


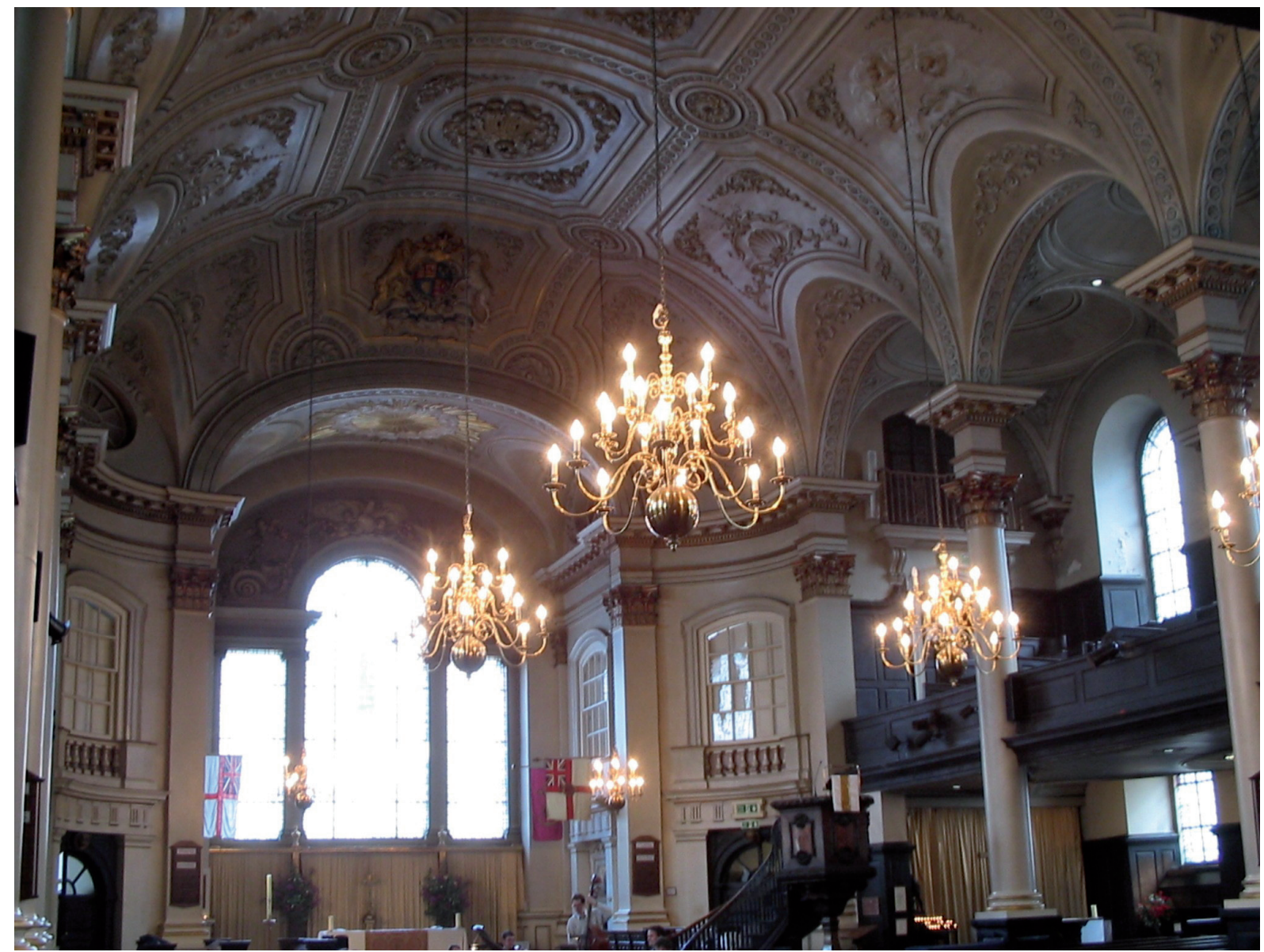

Fig. 2. Intérieur de St.-Martin-in-the-Fields à Londres avec son plafond décoré par Giovanni Battista et son fils Giuseppe Artari en 1722-26 (photographie de l'auteur).

ministre Sir Robert Walpole, mais aussi très probablement le travail de James Kent et Colen Campbell ${ }^{32}$.

Les historiens d'art qui ont analysé les œuvres des stuccatori italiens se sont essentiellement concentrés sur les individualités des artistes et ont d'abord considéré les artefacts, les dessins et les sources écrites concernant leurs travaux. Par conséquent, les artisans du stuc ont été replacés dans leur milieu artistique plus que dans leur environnement socio-économique ${ }^{33}$. Les vies des stuccatori les plus éminents tels que Paolo et PietroNatale Lafranchini, actifs en Angleterre entre 1730 et les années 1750, ont été reconstituées à partir de leur

32 Colen Campbell était l'auteur de Vitruvius Britanicus publié entre 1715 et 1725 .

33 Le travail de mise à jour le plus complet sur les stuccatori italiens de I'Angleterre du XVIII siècle est l'article de Alastair Laing, "Foreign decorators and plasterers in England", dans Hind C. éd., The Rococo in England. A symposium, Londres, Victoria and Albert Museum, 1986, pp. 21-45. apprentissage, de leur production et d'associations artistiques $^{34}$. Paolo Lafranchini a appris la plus grande partie de son répertoire ornemental de Carlo Mario Pozzi, un stuccatore majeur de la première moitié du XVIII ${ }^{e}$ siècle et auteur de l'Artis Sculptoriae Paradigmata, une collection de dessins de modèles de plafonds publiée en $1708^{35}$. Le travail des frères Lafranchini est souvent catégorisé comme un exemple de Baroque italien et du premier Palladianisme. Cependant,

34 Voir plus particulièrement Palumbo-Fossati C., Gli stuccatori ticinesi Lafrachini in Inghilterra e in Irlanda nel secolo XVIII, Lugano, Fondazione Ticino Nostro, 1982.

35 McDonnell J., Irish Eighteenth-Century stuccowork and its European sources, Dublin, The National Gallery of Ireland, 1991, p. 1. Selon Palumbo-Fossati, les Lafranchini étaient éduqués et parfaitement au courant des thèmes décoratifs mythologiques et antiques. Les motifs iconographiques contemporains pouvaient être étudiés d'après les originaux (en Italie ou pendant leurs voyages) ou à partir d'ouvrages comme l'/conologia overo descrittione dell'imagini universali cauate dall'Antichita et da altri luoghi de Cesare Ripa (1593; rééditée par Piero Buscaroli, 1988), ou les Emblemi par Andrea Alciati (Diverse imprese accommodate a diverse moralità... Tratte da gli emblemi dell'alciato, 1549). Palumbo-Fossati C., Gli stuccatori ticinesi Lafrachini in Inghilterra e in Irlanda nel secolo XVIII, Lugano, Fondazione Ticino Nostro, 1982, pp. 38-39. 
la compréhension de la production artistique des Lafranchini ne peut pas être complète sans référence à la « communauté » plus large des artisans du stuc actifs au début du XVIII siècle. La relation entre les Lafranchini et Gibbs est peu surprenante si I'on songe que Gibbs s'est formé à Rome au début du XvIII siècle auprès de Carlo Fontana, sans doute I'un des architectes natifs du Tessin les plus éminents ${ }^{36}$. L'association personnelle entre Gibbs et Fontana renforce le lien géographique entre Fontana et les nombreux stucateurs admirés par Gibbs, parmi lesquels se trouvent les Lafranchini, Giovanni Bagutti et Giuseppe Artari ${ }^{37}$.

Comme I'a souligné Shearer West, les Italiens étaient reconnus comme possédant un don particulier pour le stuc et la décoration architecturale illusionniste ${ }^{38}$. Ce « don particulier » était le résultat de leur habileté à partager un langage esthétique commun, partiellement en accord avec le palladianisme émergeant du XVIII siècle, mais en suivant aussi un réseau de relations personnelles et d'affiliation géopolitique, artistique et culturelle commune qui reliaient différents artistes comme les Artari, Bagutti, Plura, Francini et Vassali ${ }^{39}$. Cette « relation subtile » qui se traduit dans les choix esthétiques de différents stuccatori montre aussi une autre caractéristique des liens qui les unissaient. Leur " communauté » n'était pas seulement un réseau de personnes travaillant dans le même domaine, celui des arts décoratifs, et partageant une origine géographique commune. La nature "diachronique » de leurs expériences partagées, de leur répertoire et de leurs talents décoratifs communs, est une caractéristique qui dépend clairement de leur biographie individuelle, mais en même temps transcende l'expérience de chaque individu. Les Lafranchini, par exemple, arrivent en Angleterre en 1735 seulement, un quart de siècle après

36 Donati U., Breve storia di Artisti Ticinesi, Bellinzona, A. Salvioli, 1936, pp. 101-104; McDonnell J., Irish eighteenth-century Stuccowork and its European sources, Dublin, The National Gallery of Ireland, 1991, p. 2. McDonnell fait aussi remarquer comment les travailleurs du stuc, actifs dans l'Angleterre des années 1730 et 1740 , exerçaient une influence mutuelle sur leurs motifs décoratifs. Ibid., p. 4.

37 Moul T. et Earnshaw B., An insular Rococo: architecture, politics and society in Ireland and England, 1710-1770, Londres, Reaktion Books, 1999, pp. 5-6.

38 West S., "Introduction : visual culture, performance culture and the Italian diaspora in the long eighteenth century », dans West S., éd., Italian culture in Northern Europe in the eighteenth century, Cambridge, Cambridge University Press, 1999, p. 12. 39 Moul T. et Earnshaw B., An insular Rococo, op. cit. note 37, p. 4.
Bagutti et Plura ${ }^{40}$. Toutefois, leur travail ne se contente pas de se conformer au goût et aux choix esthétiques de l'époque mais intègre les travaux des stuccatori qui les ont précédés, une démarche sur laquelle comptent aussi leurs commanditaires.

\section{Le goût de I'Italie : gastronomie dans I'Angleterre géorgienne}

Le succès des Artari dépendait grandement du large mouvement intellectuel, artistique et culturel du XVIII siècle tourné vers la redécouverte des Anciens et de I'Italie de la Renaissance en Europe du Nord et plus particulièrement en Angleterre. Un travail récent a donné de précieux indice sur le rôle joué par l'Italie dans I'histoire de l'architecture, de l'art et de la musique. Les artistes, sculpteurs, scientifiques, chanteurs d'opéra, literati et macaroni italiens contribuèrent à la formation de l'Angleterre géorgienne ${ }^{41}$. Le Grand Tour faisait partie intégrante de l'éducation d'un gentleman, censé apprendre le goût italien, l'art l'architecture et la littérature classique ${ }^{42}$. Des philosophes comme Baretti, Cavallo et Cipriani, des peintres tels que Canaletto, les literati et plus d'une centaine de musiciens italiens peuvent être repérés dans I'Angleterre des Lumières du XvIII' siècle. La demande de l'Angleterre pour tout ce qui était italien était sans fin. Bien que le Grand Tour fût la prérogative de quelques personnes très aisées, la recherche de raffinement était très intense parmi ces classes qui cherchaient à grimper dans l'échelle sociale. Les quelques visiteurs revenus de l'Italie soutenaient leur position sociale en répandant le goût italien, et ainsi renforçaient leur prééminence culturelle. La recréation de la Rotonda palladienne à Chiswick par Lord Burlington est peut-être la plus célèbre des interprétations anglaises de la culture matérielle italienne au XVIII' siècle ${ }^{43}$.

La contribution artistique, intellectuelle et culturelle de I'Italie à la "société policée » de l'Angleterre du XVIII siècle est un sujet de plus de recherches que

\footnotetext{
40 Ibid., p. 5.

41 West S., «Introduction », op. cit. note 38, pp. 1-5.

42 Wilton A. et Bignamini I., Grand Tour : the lure of Italy in the eighteenth century, Londres, Tate Gallery Publishing, 1996.

43 Harris J., The Palladian revival : Lord Burlington, his villa and garden at Chiswick, New Haven, Yale University Press, 1994 ; Denman Kingsbury P., 'So noble a fabrick'. Chiswick : Lord Burlington's architectural theory and practice, Ann Arbor, MI : UMI, 1996.
} 
l'impact de ce pays sur la consommation et la vie matérielle. Une préférence latente pour la culture, contre la culture matérielle, a oblitéré le rôle de l'Italie dans l'élaboration des choix matériels et des pratiques de consommation ${ }^{44}$. L'Italie est entrée dans la maison géorgienne à travers une grande variété de supports allant des caractéristiques architecturales aux peintures, partitions, livres, estampes et verres vénitiens. Au cours du XvIII siècle, tous ces produits devinrent de plus en plus communs dans les intérieurs des élites britanniques, aidant à former un nouvel environnement pour le consommateur en devenant une partie du " mode de vie » britannique. Dans ce cas, la raisonclé de l'existence et du succès des communautés étrangères est la demande. Ce n'est pas l'élément étranger et exogène qui contribue aux transformations économiques, sociales et culturelles d'un autre pays mais le contexte lui-même qui requiert la présence active d'influences étrangères pour poursuivre des stratégies de développement économiques et de progrès culturels.

L'un des domaines spécifiques de la culture matérielle et des pratiques sociales à travers lesquels I'Italie profite de la reconnaissance européenne est I'art culinaire. L'Italie a été le centre d'élaboration de la gentilezza dans les habitudes alimentaires depuis la Renaissance, quand la vaisselle de céramique et de verre fut de plus en plus préférée aux objets métalliques. Cette vaisselle permet une meilleure présentation y compris celle des aliments et des boissons et est censée améliorer le goût de la nourriture. Dès le Xvıl siècle, les noblesses de France et d'Angleterre ont adopté les plats de majolique et les verres de cristal pour rivaliser dans leur croissante sophistication de la table ${ }^{45}$. Avant le dernier quart du $\mathrm{xv} \mathrm{I}^{\mathrm{e}}$ siècle, la verrerie de luxe était rare dans les cultures matérielles anglaise et hollandaise. Ces deux pays, indépendamment de leur prospérité économique grandissante, n'avaient pas les talents techniques et les connaissances matérielles pour produire autre chose que de la vaisselle domestique de basse qualité. Les choses commencent à évoluer à Amsterdam avec l'arrivée d'un Italien du nom de Obizzo qui ouvre une boutique de verrerie en 1597. Le verre " à la façon de Venise » est synonyme de la plus haute qualité en Angleterre aussi, où un autre émigré vénitien

44 Cfr. Grassby R., « Material culture and cultural history », Journal of interdisciplinary history, 35-4, 2005, pp. 591-604.

45 Patrick McCray W., Glassmaking in Renaissance Venice: the fragile craft, Aldershot, Ashgate, 1999, p. 82. en commence la production dès $1549^{46}$. Ces épisodes sont mieux connus pour leur transfert de précieuses habiletés vers l'Europe du Nord que pour la migration d'artisans qualifiés. La plupart des verriers émigrant de Venise et Murano vers Londres reviennent dans leurs terres natales au bout de quelques années, par crainte de la peine de mort imposée par la République, et rejetés hors du marché par les producteurs locaux.

Ce qu'il est important de souligner ici est la façon dont les produits et les pratiques sociales qui leur sont associées ont finalement été identifiés au milieu culturel italien, dépassant la fragmentation politique de la péninsule et définissant une notion archétypale de la culture de la consommation italienne. Ceci reste un important facteur, qui, bien qu'il ait été reformulé au cours des siècles suivants pour s'accorder aux contextes particuliers, a permis aux Italiens de profiter d'une « domination » esthétique et culturelle supposée. ॥ serait faux de croire que l'influence du goût italien et de la cognoscentia esthétique est confinée à la création et à la consommation d'artefacts. La cuisine italienne, au milieu de bien d'autres produits italiens, exerce une grande influence dans le consumérisme britannique $\mathrm{du}$ XVIII ${ }^{\mathrm{e}}$ siècle. Les pratiques gastronomiques et la consommation se situent entre le commerce et le savoirvivre $^{47}$. Comme beaucoup d'historiens de la nourriture I'ont observé, la cuisine méditerranéenne a de l'allure, au-delà du seul point de vue culinaire ${ }^{48}$. En Angleterre, vermicelli, macaroni, olives, huile et vinaigre, citrons et oranges, raisins, anchois, parmesan et champignons séchés font partie d'une culture britannique du dîner particulière. Tout comme l'art et la musique, la cuisine italienne est appréciée non seulement pour son goût et sa qualité mais aussi pour ce à quoi elle est culturellement associée. Ceux qui peuvent se permettre à leur table des douceurs italiennes viennent souvent de l'élite, celle-là même qui apprécie la littérature italienne et a établi des contacts avec des Italiens, en Italie comme en Angleterre.

La relation entre musique, théâtre et cuisine n'est pas seulement une relation de causalité. Elle est exploitée avec profit par les Italiens afin de gagner les

46 Ibid., pp. 158-159.

47 Sur la signification de la nourriture dans l'histoire, voir : Super J. C., « Food and history », Journal of social history 36-1, 2002, p. 165-178.

48 Flandrin J.-L., "Introduction: the early modern period ", dans Flandrin J.-L. et Montanari M., éds., Food. A culinary history from Antiquity to the present, Chichester, Columbia University Press, 1999, pp. 359-362. 


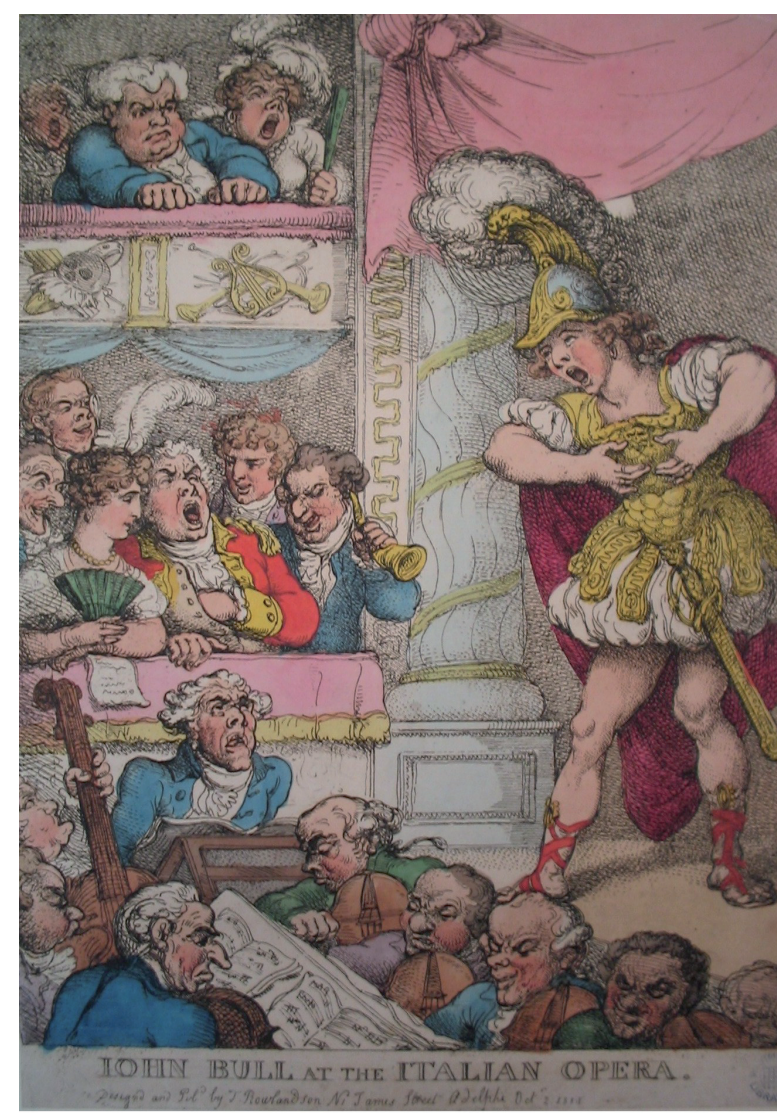

Fig. 3. « John Bull à I'Opéra italien » (reproduction autorisée par la City of Westminster - City Archives Centre, Box 63, no. 27)

faveurs d'une clientèle étrangère. De même que la cuisine, la musique et le théâtre italiens bénéficient au XVIII ${ }^{e}$ siècle d'un succès paneuropéen. L'Opera Italiana et la Commedia dell'Arte sont deux des manifestations de l'influence du « son » italien sur les cours et sociétés européennes (cf. fig. 3). Le phénomène s'étend jusqu'à générer une classe bien définie et nombreuse de musiciens et de chanteurs italiens travaillant de façon plus ou moins permanente hors de l'Italie. Le chanteur Filippo Balatri en Russie, à la fin du xvıle siècle, le musicien Domenico Scarlatti au Portugal dans les années 1720 ou Margherita Gualandi dans les années 1720 et 1730 en Europe continentale et en Angleterre ne sont que quelques cas célèbres de ce vaste phénomène culture $^{49}$. Et la bonne cuisine a souvent accompagné

49 Schlafly D. L., "Filippo Balatri in Peter the Great's Russia », Jahrbücher für Geschichte Osteuropas, 45-2, 1997, pp. 181-198; Surian E., "Domenico Scarlatti e la migrazione di musicisti italiani in Portogallo », Estudos ialianos em Portugal, 51-3, 1988-90, pp. 223-234; Freeman D. E., « An $18^{\text {th }}$-Century singer's commission of 'Baggage' arias », Early music, 20-3, 1992, pp. 427-433. Voir aussi Tatti Maria S., « Gente di teatro e di musica italiana a Parigi nel 1789 », Revue des études italiennes, 38-1/4, 1992, pp. 95-103. le chant de haute qualité. Lady Wortley Montague souligne dès 1711 I'association entre l'opéra italien et les vendeurs de produits italiens. Elle recommande à un ami d'aller chez Corticelli, un magasin renommé dans Suffolk Street, dans le West end de Londres, après dîner puisque « Mme Corticelli ou moi-même vous présenterons à Signora Checa, qui loge ici, et pendant qu'elle chante parler très doucement ne sera pas un mince hommage $\mathrm{e}^{50} »$. Conversation, musique et démonstration d'attitudes policées sont toutes présentées dans le cadre du magasin italien.

Ces magasins italiens sont d'élégantes boutiques Iondoniennes que possèdent des Italiens et où I'on vend toutes sortes de produits alimentaires « italiens » tels que parmesan, huile d'olives, anchois, pâtes sèches mais aussi saucisses, mangues et fruits exotiques ${ }^{51}$. Ce que les magasins italiens vendent est un ensemble très complexe de produits qui rappellent, en termes de qualité et de quantité, I'expérience du Grand Tour. La « Old Italian Warehouse » du ligurien Bartho Valle à Haymarket au milieu du XvIII ${ }^{\text {e }}$ siècle par exemple, offre une identité italienne claire dans ses références symboliques aux orangers et aux jarres d'huile (cf. fig. 4$)^{52}$. Valle est sans doute le mieux connu parmi un petit groupe de marchands italiens tenant boutique dans le Londres géorgien. Des détails de la vie de Valle en Angleterre, ou de celles d'autres détaillants

50 "Letter 13 February 1711 », dans Halsband R., éd., The Complete letters of Lady Wortley Montague, Oxford, Clarendon, 1965, vol. i, p. 75.

51 Sur ce sujet voir Riello G., "The taste of Italy: Italian businesses and the culinary delicacies of Georgian London », London Journal, 26-2, 2005. Voir aussi Allen B., «Foreign flavours: the Italian warehouse and its near relations in England, 1720-1880 », dans Spicing up the palate. Studies of flavourings - Ancient and modern, Prospect Books, 1992.

52 Néanmoins, l'entête de facture de 1735 de Valle inclut des produits tels que «Fine Italian artificial Flowers. Cyprus wine, Frontiac ditto. St Loran ditto. The best Artemino ditto. Chianti and Carmignano ditto. The best Italian Rozazolis from Bologna and Nizza, different Sorts. Maraschino of Corfu, and Huil de Venus. Fine Provence Oil. Lucca and Florence ditto... Italian Bolognia Sausages and Mortadelle. Westphalia and Bayona Hams, Dutch Beef... Small Roman Capers. Capucin and Genoua ditto. Pickl'd Mushrooms, Italian Lintuls » et ainsi de suite. BM, 89.159: « Billhead of 'B. Valle and Brother at the Old Italian Warehouse ». Moins d'un tiers des produits de Valle a une provenance italienne claire. Vingt pour cent de ces articles peuvent être qualifiés d'origine française. Ces produits de base étaient complétés par des spécialités hollandaises, allemandes et espagnoles. À peu près treize pour cent des biens à vendre sont des essences et enfin quatre pour cent viennent d'Asie. 


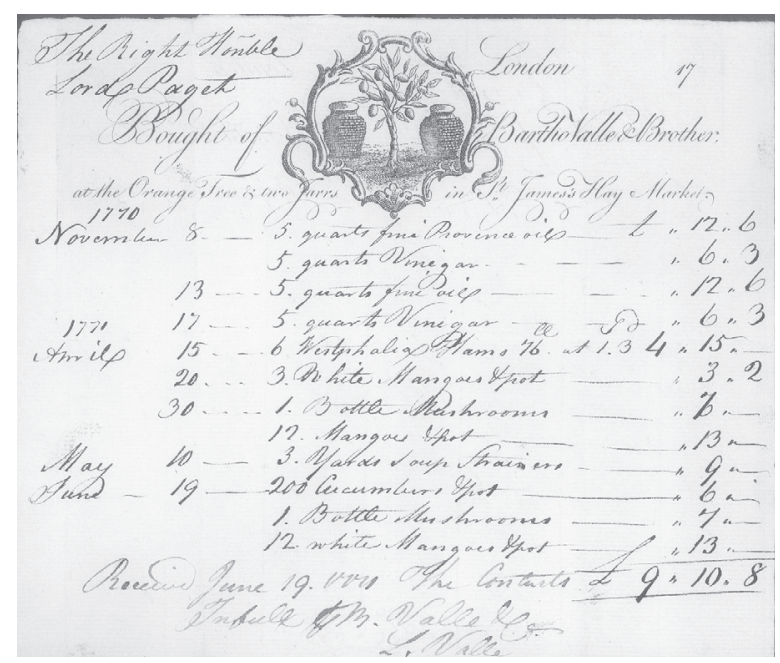

Fig. 4. Facture de Bartho Valle pour Lord Paget, 19 juin 1770 (reproduction autorisée par la Corporation of London - London Metropolitan Archives, ACC/446/H23/83)

d'alimentation italienne actifs au milieu du xvIII siècle, peuvent s'entr'apercevoir à travers l'autobiographie du florentin Filippo Mazzei qui vécut à Londres entre 1754 et 1772 et fut lui-même pendant un temps propriétaire d'un magasin italien.

Au début de 1760, Filippo Mazzei reçoit une lettre d'Andrea Bonducci, un ami proche, qui vit à Florence. Bonducci écrit quatre ans après le départ de Mazzei pour Londres, initialement comme médecin et plus tard comme professeur privé d'Italien pour les riches aristocrates de la métropole. Mazzei est très impressionné par une observation de Bonducci dans sa lettre. Bonducci et Mazzei semblent chercher une méthode facile mais pas trop compromettante, de faire de l'argent. Mazzei se débat pour boucler ses fins de mois et se flatte de mener une vie de gentleman, entretenant une maison dans Greek Street près de Soho Square et se mêlant à cette élite métropolitaine à laquelle il enseigne I'Italien. Ses gages comme enseignant ne suffisent pas pour maintenir un train de vie équivalent à celui de la noblesse. L'autobiographie de Mazzei révèle de précieux indices sur sa difficile position sociale et financière ${ }^{53}$. Dante et Pétrarque font son pain quotidien. Toutefois, la lettre de Bonducci suggère que I'Italie peut subvenir à d'autres besoins, plus « savoureux » et prosaïques. II professe, d'un ton

53 Mazzei F., Memoirs of the life and peregrinations of the Florentine Philip Mazzei, New York, 1942. Le texte original a été publié en italien sous le titre Memorie della vita e delle peregrinazioni del Fiorentino Filippo Mazzei, Lugano, 1846. plutôt provocateur, « [qu']il est beaucoup plus fréquent pour Mes Seigneurs de prendre soin de leur cellier que de leur bibliothèque, et cela est du au fait qu'ils aiment boire bien plus qu'ils n'aiment lire ${ }^{54} »$. Ces mots ont un effet profond sur Mazzei. II commence à voir le potentiel de la nourriture et des boissons sur le considérable marché de la culture italienne. Un goût raffiné ne s'acquiert pas seulement dans les bibliothèques et les théâtres, mais s'apprend aussi autour d'un dîner.

Mazzei commence à vendre des produits alimentaires italiens en usant de sa position sociale dans la société londonienne pour faire des transactions avantageuses, vendant toutes sortes de produits importés à ses amis et connaissances. Au départ, il n'y avait pas d'entreprise au centre de sa nouvelle activité économique mais un cercle d'intellectuels londoniens bien connus, de femmes tenant salon et de figures littéraires. Parmi ses amis les plus proches se trouvent des personnalités comme les fameux docteurs Samuel Sharp et William Bromfield, Matthew Maty (le bibliothécaire du British Museum et secrétaire de la Royal Society), I'anatomiste John Hunter, Pietro Paolo Celestia (le ministre de la République de Gênes à Londres), le poète David Mallet et le jeune Edward Gibbon à qui Mazzei enseigne l'Italien. Mazzei cultive soigneusement non seulement ses relations londoniennes mais aussi ses contacts et amitiés en Italie avec la « haute sphère » de l'élite politique et culturelle $\mathrm{e}^{55}$.

Mazzei se voit comme un médecin et tient à apparaître devant ses amis et connaissances comme un homme entreprenant plus que comme un entrepreneur. Il considère ses activités commerciales comme un moyen de faciliter d'importantes relations culturelles et sociales, qu'il cultive afin de devenir un membre à part entière de la "société » à Londres comme à Florence. II est clair qu'il est conscient des profits du commerce alimentaire. Un voyage en Italie en 1760-1761 est I'occasion de renforcer les contacts commerciaux avec des producteurs. Là encore, il se sert de son cercle d'amis pour son profit économique. L'un des cousins de Mazzei à Leghorn le présente à Santi Bargellini, I'un des principaux producteurs de vin et plus tard son plus important fournisseur. À travers d'autres relations, il acquiert une quantité substantielle de vin de Cartei qu'il met plus tard en bouteille à Londres. Enfin il va goûter de l'huile d'olive, une partie de la production

54 lbid., p. 163.

55 Becattini M., Filippo Mazzei, mMercante italiano a Londra (1756-1772), Prato, Pentalinea, 1997, p. 18. 
de Filippo Neri, le frère du célèbre avocat Pompeo Neri. Sur le chemin du retour vers Londres, il achète de grandes quantités de Champagne en France. De retour à Londres : "Par Lady Talbot, j'ai vendu en un jour, à ses amis, tout mon premier approvisionnement de champagne, avec un bénéfice de soixante-quinze guinées. J'ai vendu bien d'autres produits en gros, de I'huile de Lucques et des collines de Pise... mise dans des pots de terre et dans des caisses ; des fromages de Lodi et Codogno (que j'ai aussi exportés à mon propre compte vers les Indes Occidentales); divers produits de la Provence; des articles manufacturés de Lyon, qui m’ont été amenés par bateaux via Leghorn ; des farineux ; des greffons de citronnier ; des fruits candis et d'autres produits génois ; et des variétés de soie de Florence, surtout celles utilisées pour les doublures ${ }^{56}$. »

En 1764, Mazzei ouvre finalement un magasin italien dans New Bond Street, le centre de la société policée, qui, selon Mazzei, est parfait pour tenir boutique puisque que c'est : « un lieu fréquenté et très actif, similaire à la via Calzaioli à Florence, avec la différence que dans la via Calzaioli deux voitures peuvent à peine se croiser alors que dans New Bond Street trois peuvent se déplacer confortablement côte à côte. De plus, il y a des trottoirs des deux côtés de New Bond Street, chacun large de plus de six pieds ${ }^{57}$. »

Bien que Mazzei ait été prudent dans le choix de son local et dans la gestion de la nouvelle boutique, il découvre rapidement que le monde des boutiques italiennes dans le Londres géorgien est livré à une forte concurrence. Un petit groupe d'exploitants italiens et britanniques se battent sur le marché pour assurer leur position.

L'année précédant l'installation de Mazzei, ce dernier est poursuivi en justice par un certain Angelo Bernardi, un Italien possédant une boutique dans Frenchurch Street dans la City de Londres ${ }^{58}$. Bernardi prétend que Mazzei et quelques autres Italiens portent activement dommage à ses intérêts commerciaux en répandant la rumeur qu'il fait face à une banqueroute. L'épisode, de peu de conséquences pour Mazzei à l'époque, réapparaît quelques années plus tard sous une forme un peu différente. Mazzei mentionne dans

56 Mazzei F., Memoirs, op. cit. note 53, pp. 125-26. L'auteur a en partie modifié cette citation en suivant le texte italien.

57 Ibid., p. 128.

58 Becattini, Filippo Mazzei, op. cit. note 55, p. 18; TNA (The National Archives, London), C/12/1502/27 : «Bernardi v. Mazzei, 1771 ». De nombreux états des marchands qualifient Angelo Bernardi de "Merchant». son autobiographie qu'en septembre 1768 il ouvre une deuxième boutique dans Haymarket. C'est la boutique que Mazzei a voulue pendant des années. Elle est en fait en face de I'Opera Theatre ou « Teatro dell'Opera Italiana » et proche du Coffee hoouse du Prince d'Orange dans Haymarket ${ }^{59}$. La combinaison haute culture-négoce est évidente. C'est le point focal de la communauté italienne, les célèbres castrati, la musique et la mode ${ }^{60}$. Cette expansion assure deux boutiques pour Mazzei, toutes les deux avec des situations en vue, ce qui le place clairement au centre d'un large réseau culturel italien. Sa quête pour entrer dans la bonne société a finalement porté ses fruits après plus d'une décade.

Comme tout document personnel, I'autobiographie de Mazzei peut difficilement être pris pour argent comptant. Dans les pages dédiées à sa vie londonienne il n'est pas fait mention de l'importante procédure légale dans laquelle il se trouvait en 1769. Ce n'est pas par chance que Mazzei trouve I'emplacement idéal pour sa nouvelle boutique. En 1768, c'est Mazzei lui-même qui poursuit en justice le propriétaire, le même Angelo Bernardi qui l'a dénoncé en $1763^{61}$. Mazzei a acheté au marchand génois Filippo Nardi une traite faite par Bernardi. Apprenant que Bernardi a demandé au frère de Nardi de repousser le paiement d'une autre facture, Mazzei demande à ce qu'elle soit payée immédiatement, mais Bernardi n'en a pas les moyens. La situation débouche sur la déclaration de banqueroute de Bernardi. Le contenu et le bail de la boutique sont achetés par James Frog, un huilier de Fleet Street. Après que Bernardi est sorti de prison, il réalise que le principal bénéficiaire de ses déboires a été Mazzei, qui a acheté à James Frog la plupart de ses biens et le bail de la boutique ${ }^{62}$.

Les Chancery Masters' Exhibits ne donnent aucune preuve que Mazzei ait été poursuivi. Son magasin est aussi prospère que possible, bien que

59 Kent's directory for the year 1768, 114 et 116 ; Baldwin's new complete guide, 1768, pp. 140-141.

60 Voir plus particulièrement Petty F. C., Italian opera in London, 1760-1800, Ann Harbour, UMI Research Press, 1980; Rosselli J., Singers of Italian ppera: the history of a profession, Cambridge, Cambridge University Press, 1992.

61 TNA, C/12/1502/27 : «Bernardi v. Mazzei, 1771 ». Il est toutefois difficile d'estimer la réputation de Bernardi. D'autres témoignagnes suggèrent qu'il était un marchand peu scrupuleux. TNA, C12/1007/4 : «Bernardi vs Moretti, 1763 »; E133/21/11: «Bernardi vs Moretti, 1764 ».

62 TNA, C/12/1502/27. 
sa position dans la société ait été entachée de façon permanente par l'affaire Bernardi. L'association entre culture et négoce que Mazzei a nourrie et pondérée pendant des années détruit maintenant sa position sociale. Ce qui a été appelé scandale a été largement répandu par l'écrivain, philosophe et bon vivant italien Carlo Francesco Badini dans un ouvrage intitulé /I Vero Carattere di Giuseppe Baretti publié à Londres en $1770^{63}$. Le prologue de son livre est une attaque véhémente contre Mazzei, à l'époque ami proche de Giuseppe Baretti, I'un des ennemis les plus méprisés de Badini. Selon Badini, Mazzei est un parvenu d'humble origine qui n'est rien de plus qu'un boutiquier, mais qui s'est mêlé d'affaires commerciales douteuses et a fait des profits aux dépens de ses amis italiens et britanniques ${ }^{64}$.

Les Mémoires de Mazzei sont un point de vue biaisé mais unique sur les magasins italiens de Londres au XVIII siècle. Elles présentent une vision particulière d'un groupe de gens qui partagent une nationalité et une profession commune mais qui ne sont sûrement pas une communauté. Si Mazzei est vraiment représentatif d'un groupe d'Italiens vendant au détail de coûteux produits alimentaires à la haute société de Londres, son expérience montre comment le réseau externe d'amis et de connaissances, en Angleterre comme en Italie, est plus important que la coopération entre nationaux à Londres. Un élément second mais tout aussi important de I'histoire de Mazzei est la relation entre profit commercial et statut social. L'Italie n'est pas une terre mère lointaine et oubliée, comme elle le fut pour de

63 Badini C. F., Il vero carattere di Giuseppe Baretti Pubblicato per amor della virtù calunniata: per disinganno degli Inglesi e in difesa degli Italiani, Londres [?], 1770, p. 11.

64 La position de Mazzei était encore plus compromise par le feu qui détruisit sa boutique de Haymarket moins d'un an après son acquisition. C'est un épisode peu clair de la vie de Mazzei. Selon Mazzei la boutique " was set on fire » (« on a mis le feu à la boutique »). Mais des compatriotes et des concurrents ont informé une personne de la compagnie d'assurance que la valeur du stock de Mazzei était de moins de 400f, alors qu'il était assuré pour 1.000f, et que l'on a ordonné à l'un des serviteurs de Mazzei de mettre le feu à la maison. Mazzei F., Memoirs, op. cit. note 53, p. 168; Guildhall Library (GL), Mss 8666, vol. 21, f. 20, 22 et 28: "Hand in Hand General Minute Book » (1926 septembre 1769); GL, MS 11934, vol. 6, 11 octobre 1769 : "Sun Insurance Office: Town Committee Minute Book»; GL, MS 12019, vol. 3, f. 46 : " Sun Fire Office : General Cash Book» (29 September 1769); GL, Mss 14022, vol. 14, 11 octobre 1769 : «Union Fire Office : Board and Committee Minutes». Tous ces documents sont reproduits dans Becattini M., Filippo Mazzei, op. cit. note 55, pp. 85-89. nombreux émigrants du XIX $x^{e}$ et $x X^{e}$ siècle, mais la source même de son statut culturel à l'étranger.

\section{Conclusion : le legs de I'Italie}

Stuc et produits alimentaires ne sont que deux domaines dans lesquels l'Italie devint aux $x v I^{\mathrm{e}}$ et XVIII siècles synonyme de haute qualité et de goût. Des analyses similaires peuvent être faites pour la verrerie, les petits objets de cuir, carrelage mais aussi pour l'architecture, peinture, etc. La liste est longue et peut être résumée de façon synthétique par ce que I'on appelle « industrie légère et services », dont la contribution à l'économie de l'Italie d'aujourd'hui est toujours considérable. II semble y avoir un unique sens de la continuité entre les aires d'activités économiques, dans lesquelles la péninsule italienne excelle à la Renaissance, et au début de la période moderne, et sa spécialisation industrielle contemporaine. L'un des buts de cet article a été de souligner à travers deux cas précis le développement à long terme de ce que I'on nomme le «made in Italy ». Cependant, il ne $s^{\prime}$ agit pas seulement de reconnaître des trajectoires de développement fondées sur des productions spécialisées spécifiques. Une question émerge de la discussion sur les produits italiens du XVII ${ }^{e}$ ou XVIII ${ }^{e}$ siècle : celle de leur identité spécifique, plus particulièrement lorsqu'ils sont négociés, vendus et consommés hors des frontières nationales de l'Italie.

La question devient alors pourquoi et comment I'Italie a-t-elle élaboré une telle identité et a su la reproduire au cours des siècles ? La réponse n'est pas directe puisque différents cas suggèrent des faisceaux variés d'explications. Les stuccatori du Tessin, par exemple, montrent leur capacité à poursuivre une production spécialisée pendant plusieurs générations grâce à des liens particuliers avec les architectes et à des ensembles de pratiques (comme le recrutement des apprentis) qui étendent leur travail aux générations suivantes. Les travailleurs du stuc du Tessin sont aussi emblématiques de la participation active de travailleurs italiens talentueux aux contextes culturels et économiques de l'étranger, car ils montrent comment le processus de définition de la « distinction » italienne débute et se développe hors d'Italie depuis le commencement de la période moderne. Les marchands de produits alimentaires italiens dans le Londres du XVIII siècle montrent plutôt un sens moins cohérent 
de la « communauté ». Leur compétition commerciale s'entremêle avec l'existence de différents groupes et positions culturelles dans la société britannique. Le monde du théâtre italien est, dans ce cas, central dans la construction d'une culture culinaire italienne qui fournit à une clientèle anglaise « recherchée » I'opportunité de traduire dans ses comportements quotidiens de consommateurs ses notions érudites sur I'architecture et le goût artistique. 